\title{
Avaliação dos fatores associados com a candidíase bucal em pacientes do Serviço de Estomatologia do Hospital São Lucas - PUCRS
}

\author{
Evaluation of associated factors with oral candidiasis in patients from \\ Stomatology Service of São Lucas Hospital - PUCRS
}

\author{
Fernanda Gonçalves Salum * \\ Gabriela Botelho Martins * \\ Lisandrea Rocha Schardosim * \\ Nadiesca Maria Lazzari Miotto* \\ Maria Antonia Zancanaro de Figueiredo**
}

\section{RESUMO}

A candidíase bucal é uma doença infecciosa fúngica causada principalmente pela Candida albicans, cujas manifestações clínicas podem estar vinculadas a fatores locais e sistêmicos. 0 objetivo deste estudo foi avaliar a associação entre fatores como tabagismo, alcoolismo, anemia, diabete, antibioticoterapia, corticoterapia, radioterapia, quimioterapia e soropositividade para o HIV com as diferentes formas clínicas da candidíase bucal. Foram analisados 303 prontuários de pacientes com candidíase bucal, atendidos no período de 1990 a 2000, dos quais foram excluídos aqueles que apresentavam candidíase associada ao uso de prótese. Dos 90 prontuários inclú́dos no estudo, observou-se que 62 pacientes $(68,9 \%)$ apresentavam uma das formas clínicas da doença e que 28 possuíam associação entre duas $(26,7 \%)$ ou três $(4,4 \%)$ formas. A apresentação clínica mais prevalente foi a eritematosa $(38,9 \%)$, seguida pela pseudomembranosa $(37,8 \%)$, leucoplásica $(27,8 \%)$, queilite angular $(21,1 \%)$ e glossite romboidal mediana (GRM) (10\%). A forma leucoplásica foi associada com o tabagismo $(p=0,01)$, enquanto a candidíase eritematosa teve associação com anemia $(p=0,04)$. A candidíase pseudomembranosa apresentou relação estatisticamente significante com antibioticoterapia $(p=0,01)$, radioterapia $(p=0,05)$, quimioterapia $(p=0,02)$ e soropositividade para o HIV $(p=0,01)$. A queilite angular e a GRM não foram estatisticamente associadas aos fatores estudados. Concluiu-se que existe associação entre a forma de apresentação clínica da candidíase bucal e determinados fatores locais e sistêmicos.

\section{PALAVRAS-CHAVE}

\section{INTRODUÇÃO}

A candidíase bucal é uma infecção fúngica oportunista causada pela Candida sp., principalmente a espécie albicans. O microrganismo é um habitante comensal da boca, do trato gastrintestinal e da vagina de pessoas não afetadas clinicamente, portanto sua mera presença não é suficiente para produzir a doença. Uma série de fatores locais e sistêmicos permite que o microrganismo se instale e manifeste seu potencial patogênico. Entre eles podemos citar os antibióticos, drogas imunossupressoras, xerostomia, distúrbios endócrinos e imunológicos, AIDS, radioterapia, idade precoce ou muito avançada, períodos pós-cirúrgicos, tabagismo e tipo de dieta (BUDTZ-JÖRGENSEN 1990; OKSALA , 1990; CANNON; CHAFFIN ${ }^{2}, 1999$ ).

Existem várias formas clínicas da doença, sendo a candidíase eritematosa ou atrófica crônica a mais comum, estando principalmente associada ao uso de próteses totais ou parciais removíveis. Outras formas de apresentação da candidíase bucal são a pseudomembranosa, leucoplásica, queilite angular $e_{20}$ glossite romboidal mediana (SHAFER $^{200}$ et al., 1987; NEVILLE ${ }^{3}$ et al., 1998).

Baseado na literatura disponível questiona-se a existência de uma associação entre a presença de fatores locais e

\section{Candidíase bucal; etiologia; fatores de risco} sistêmicos e a forma de manifestação clínica da candidíase bucal. Assim, este estudo retrospectivo tem o objetivo de determinar a relação entre fatores sistêmicos e locais com as diferentes formas clínicas de candidíase bucal em pacientes atendidos no Serviço de Estomatologia do Hospital São Lucas da PUCRS.

\section{REVISÃO DA LITERATURA}

Múltiplos fatores locais e sistêmicos estão relacionados à infeçção bucal por Candida sp. Segundo Cox (1983), a terapia antibiótica promove um aumento da glicose salivar por reduzir a competição bacteriana, o que favorece a aderência de fungos às células epiteliais da mucosa bucal. A virulência da $C$. albicans é significativamente aumentada em meios hiperglicêmicos. Dessa forma, o uso prolongado de antibióticos é um fator predisponente à infecção por essa levedura (BUDTZ-JÖRGENSEN", 1990).

Pacientes com deficiência imunológica, como aqueles submetidos a tratamentos quimio e radioterápicos, têm alto risco de sofrerem infecção invasiva provocada por Candida sp. (SONIS et al. , 1985; HEIMDAHL; NORD ${ }^{\circ}, 1990$ ). Do mesmo modo, em pacientes infectados pelo HIV, a candidíase bucal, principalmente a forma pseudomembranosa, é uma complicação $\quad$ freqüente
HODGSON; RACHANIS). McCarthy et al. (1991) avaliaram pacientes HIV soropositivos e encontraram uma prevalência de $34 \%$ de candidíase bucal, sendo a forma eritematosa a mais freqüente. Segundo os autores, a deficiência de linfócitos $\mathrm{T}$, associada à progressão da doença, e a xerostomia causada pelo uso de drogas anti-colinérgicas e medicamentos anti-depressivos são fatores importantes no aparecimento da infecção fúngica.

Pacientes submetidos à tratamento radioterápico na região de cabeça e pescoço também apresentam um quadro progressivo de xerostomia. A redução do fluxo salivar implica num aumento da colonização por Candida sp. na cavidade bucal e conseqüente manifestação clínica da doença (RAMIREZ-AMADOR et al. 1997). De 2 acordo com o estudo de TORRES et al. ${ }^{22}$ (2002), o número de unidades formadoras de colônia (UFC) apresenta uma relação inversa ao fluxo salivar em pacientes com queixa de xerostomia.

Outro fator associado à candidíase bucal é a anemia por deficiência de ferro, pois esta causa depleção da imunidade mediada por células e favorece o aparecimento de infecções oportunistas (BUDTZJÖRGENSEN, 1990). Da mesma forma, a anemia aplástica é uma das doenças sistêmicas que predispõem às infecções fúngicas, pois afeta de modo adverso a capacidade do paciente combater esses mi-

* Alunas do Programa de Doutorado em Estomatologia Clínica da Pontifícia Universidade Católica do Rio Grande do Sul ** Professora titular do Programa de Doutorado em Estomatologia Clínica da Pontifícia Universidade Católica do Rio Grande do Sul 
crorganismos (SONIS et al. ${ }^{21}, 1985$ ).

Estudos indicam que o tratamento tópico ou sistêmico com corticosteróides ou outras drogas imunossupressoras podem predispor à candidíase bucal e têm o potencial de agravar essas infecções, quando pré-existentes (BUDTZ-JÖRGENSEN ${ }^{1}$, 1990; OLIVEIRA et al. , 1998). Da mesma forma, a candidíase crônica bucal é um problema freqüente em pacientes que estão recebendo terapia por inalação de esteróides (LAMEY; LEWIS , 1997).

Além do uso de medicação inalada e da utilização de próteses removíveis, outro fator local que pode estar relacionado à infecção por Candida sp. é o tabagismo. A candidíase leucoplásica é a forma clínica mais comumente associada a este fator, uma vez que aproximadamente 10\% das biópsias de lesões brancas da cavidade bucal mostram alterações histológicas típicas de candidíase (KOSTIALA et al. , 1979). Greenspan et al. (1992) observaram, em pacientes HIV soropositivos, uma associação entre a candidíase pseudomembranosa e o tabagismo.

\section{MATERIAIS E MÉTODOS}

A presente pesquisa se insere no paradigma quantitativo, em uma abordagem descritiva ex post facto. A hipótese baseia-se na afirmação de que existem fatores capazes de favorecer a ocorrência de candidíase bucal em suas diferentes formas de apresentação. As variáveis independentes incluem possíveis fatores predisponentes da candidíase bucal, sendo analisados neste estudo anemia, diabete, soropositividade para o HIV, o relato de consumo crônico de bebidas alcoólicas e tabaco, estar usando antibióticos, corticosteróides bem como recebendo tratamento radio e/ou quimioterápico.

Essa pesquisa foi realizada em um universo de 303 prontuários de pacientes do Serviço de Estomatologia e Prevenção do Câncer Bucomaxilofacial do Hospital São Lucas da PUCRS, atendidos no período compreendido entre janeiro de 1990 e outubro de 2000, cuja queixa principal foi diagnosticada como candidíase bucal. Desses prontuários foram excluídos aqueles que apresentavam candidíase eritematosa associada ao uso de próteses totais ou parciais removíveis. Sendo assim, foram utilizados 90 prontuários de pacientes com candidíase bucal para análise da relação entre a forma clínica da doença e os fatores predisponentes já citados.

Para avaliar estatisticamente as variáveis e suas relações com as formas clínicas da doença, foram utilizados os testes Qui-quadrado e exato de Fischer considerando menor ou igual a 0,05 .

\section{RESULTADOS}

Dos 90 prontuários analisados, 42 representavam indivíduos do sexo masculino e 48 do sexo feminino. Dentre as apresentações clínicas encontradas, observouse que 62 pacientes $(68,9 \%)$ possuíam exclusivamente uma forma da doença e 28 deles, associação de duas $(26,7 \%)$ ou três formas clínicas $(4,4 \%)$.

Conforme representado no gráfico 1 , do total de formas clínicas encontradas, $35(38,9 \%)$ eram de candidíase eritematosa; $34(37,8 \%)$ de candidíase pseudomembranosa; $25(27,8 \%)$ pacientes apresentavam a forma leucoplásica; $19(21,1 \%)$ possuíam queilite angular associada a candidíase bucal, enquanto 9 (10\%) casos eram de GRM.

A análise dos prontuários revelou que, em ordem decrescente de freqüência, os fatores locais e sistêmicos encontrados foram tabagismo $(n=53)$, soropositividade para o HIV $(n=17)$, alcoolismo $(n=13)$, anemia $(n=12)$, corticoterapia $(n=9)$, antibioticoterapia $(n=8)$, diabete $(n=5)$, quimioterapia $(n=4)$ e radioterapia $(n=3)$. Em 17 pacientes não foram encontrados os fatores previamente descritos.

A análise estatística demonstrou que para a candidíase pseudomembranosa (tabela 1), os fatores significativamente associados foram antibioticoterapia, radioterapia, quimioterapia e soropositividade para o HIV. Para a candidíase eritematosa (tabela 2), a anemia foi o único fator positivamente associado. A candidíase leucoplásica (tabela 3) encontrou-se fortemente relacionada com o tabagismo. Nenhum dos fatores estudados foi significativamente associado à presença de queilite angular e GRM.

\section{DISCUSSÃO}

Considerando que o presente estudo trata-se de uma análise retrospectiva de prontuários, a ausência de determinados dados nos mesmos, dificultou a obtenção de algumas informações. Não foi possível estabelecer a frequiência e a quantidade do consumo de bebidas alcoólicas, portanto foram considerados etilistas pacientes que ingerissem qualquer tipo de bebida alcoólica diariamente. Quanto ao consumo de tabaco, foram considerados tabagistas pacientes que relataram ser fumantes no momento do diagnóstico da candidíase. $\mathrm{O}$ tratamento com antibióticos e com corticosteróides foi considerado quando os mesmos estivessem sendo utilizados no momento do exame. $\mathrm{O}$ uso, ou não, de medicamentos anti-retrovirais em pacientes portadores do HIV não foi considerado, assim como, em pacientes diabéticos, não se reavaliou a glicemia.

As formas clínicas de candidíase mais comumente encontradas nos prontuários analisados foram eritematosa e pseudomembranosa, seguidas, em ordem decrescente pelas leucoplásica, queilite añ gular e GRM. No estudo de Miotto et al. (2003), a candidíase eritematosa foi a apresentação clínica mais encontrada, estando comumente associada ao uso de próteses totais ou parciais removíveis uma vez que a má higiene e desadaptação das mesmas favorecem a adesão dạ Candida sp. ao acrílico (RADFORD et al. , 1999). Dos 303 prontuários analisados no presente estudo, 213 casos eram de candidíase associada a próteses totais ou parciais removíveis. $\mathrm{Na}$ tentativa de identificar outros fatores locais ou sistêmicos, optou-se, nesta investigação, por excluí-los.

A candidíase pseudomembranosa pôde ser associada, nesse estudo, a diversos fatores: antibioticoterapia, radioterapia, quimioterapia e soropositividade para o HIV. A forma pseudomembranosa foi a mais encontrada por Kongkunthian et al. (2001) em pacientes soropositivos para o HIV, corroborando os resultados deste estudo. Assim como a infecção por HIV, a quimioterapia é um fator sistêmico de imunossupressão e por isso, também associado à candidíase. A radioterapia de cabeça e pescoço é um fator local, que implica em redução do fluxo salivar, favorecendo a manifestação clíniça da doença (RAMIREZAMADOR et al. , 1997). A terapia antibiótica, quando instituída, reduz a competição entre os fungos e as bactérias bucais, havendo aumento da glicose salivar e conseqüentemente favorecȩndo a proliferação da Candida sp. (COX , 1983).

A anemia por deficiência de ferro causa depleção da imunidade mediada por células favorecendo o aparecimento de infecções oportunistas (BUDTZ JÖRGENSEN, 1990). Rennie et al. (1983) observaram incidência estatisticamente significativa de candidíase crônica em ratos anêmicos, concordando com os achados do presente estudo, onde a anemia foi o único fator associado à candidíase eritematosa. Resultado contrastante, no entanto, foi encontrado por McCarthy et al. (1991) demonstrando que a forma eritematosa foi a mais prevalente em pacientes soropositivos para o HIV. Nestes pacientes a contagem de células T CD4 é considerada o segundo maior preditor para a ocorrência de candidíase.

A etiologia da candidíase leucoplásica não é completamente compreendida, pois permanece a dúvida se a Candida sp. se sobrepõe a uma leucoplasia pré-existente ou se a mesma é capaz de promover o desenvolvimento de lesões hiperceratóticas (NEVILLE et al. , 1998). Nesse estudo, observou-se forte associação da candidíase leucoplásica e o tabagismo, onde de 25 casos analisados, 24 eram pacientes tabagistas.

A queilite angular e a GRM foram as formas clínicas menos freqüentes nesse estudo, o que está de acordo com os dados da literatura (PUGLIESE et al. , 1972; FIGUEIREDO et al., 2002). Nenhum dos fatores estudados foi associado com essas formas de apresentação clínica da doença, porém, vale ressaltar, que o tamanho da amostra era pequeno e por isso o teste estatístico não foi significativo, embora revelasse valor expressivo $(\mathrm{p}=0,06)$ para a associação de queilite angular e diabete. No presente estudo as variáveis foram analisadas isoladamente, no entanto, cada paciente poderia apresentar mais de um fator de risco para o desenvolvimento de candidíase bucal, o que pode ter influenciado nos resultados obtidos. Através dos 
dados deste estudo concluiu-se que existe associação entre as formas de apresentação clínica da candidíase bucal e determinados fatores locais e sistêmicos. No entanto, são necessárias novas pesquisas no âmbito da biologia molecular para esclarecer se diferentes fatores que interfiram na virulência da Candida albicans podem determinar a forma de apresentação clínica da doença.

\section{ABSTRACT}

Oral candidiasis is an infectious disease caused mainly by Candida albicans, whose clinical manifestations can be associated with local and systemic factors. This study aimed to evaluate the association between factors like smoking, alcoholism, anemia, diabetes, antibiotic therapy, corticoid therapy, radiotherapy, chemotherapy, HIV infection with the different clinical forms of oral candidiasis. Three hundred and three records of patients with oral candidiasis were analyzed. Those records which patients presented erythematous candidiasis associated to the use of prothesis were excluded. From the 90 remaining patient records, 62 patients $(68,9 \%)$ presented one clinical form of the disease and that 28 patients presented an association of two $(26,7 \%)$ or three $(4,4 \%)$ forms. The clinical presentation of oral candidiasis that prevailed most was the erythematous $(38,9 \%)$ followed by the pseudomembranous $(37,8 \%)$, the candidal leukoplakia $(27,8 \%)$, as well as angular cheilitis $(21,1 \%)$ and median rhomboid glossitis (10\%). The candidal leukoplakia was associated to smoking $(p=0,01)$, while the erythematous candidiasis was associated to anemia $(p=0,04)$. Pseudomembranous candidiasis presented statistically significant relation to antibiotic therapy $(p=0,01)$, radiotherapy $(p=0,05)$, chemotherapy $(p=0,02)$ and HIV infection $(p=0,01)$. Angular cheilitis and median rhomboid glossitis were not statistically associated to any of the studied factors. Hence, it was found that there is an association between the form of clinical presentation of oral candidiasis and some determined local and systemic factors.

\section{KEYWORDS}

Oral candidiasis, etiology, risk factors.

\section{REFERÊNCIAS}

BUDTZ-JÖRGENSEN, E. Etiology, Pathogenesis, Therapy, and Prophylaxis of Oral Yeast Infections. Acta Odontol Scand, Stockholm, v.48, n.1, p.61-69, Feb. 1990.

CANNON, R. D., CHAFFIN, W. L. Oral Colonization by Candida albicans. Crit Rev Oral Biol Med, Boca Raton, v.10, n.3, p.359-383, 1999.

COX, F. Adherence of Candida Albicans to Buccal Epithelial Cell in Children and Adults. J Lab Clin Med, St Louis, v.102, n.6, p.960-972, Dec. 1983.

FIGUEIREDO, M. A. Z.; DUARTE, R.; LOUREIRO, M. et al. Estudo da Epidemiologia e Etiopatogenia da Glossite Rômbica Mediana nas Diferentes Faixas Etárias. Pesq Odontol Bras, São Paulo, v. 16, Suplemento (Anais da 19 ${ }^{\mathrm{a}}$ Reunião Anual da SBPq0), p.204, 2002.

GREENSPAN, D.; HILTON, J.F.; WESTENHOUSE, J.L. et al. Association Between Oral Lesions and Smoking in a Clinic Population. Int Conf AIDS, v.8, n.2, p.19-24, 1992.

HEIMDAHL, A; NORD, CE. Oral Yeast Infections in Immunocompromised and Seriously Diseased Patients. Acta Odontol Scand, Stockholm, v.48, n.1, p.77-84, Feb. 1990.

HODGSON, T.A., RACHANIS, C.C. Oral Fungal and Bacterial Infections in HIVInfected Individuals: An Overview in Africa. Oral Dis, Houndmills, v.8, Suppl.2, p.80-87, 2002.

KHONGKUNTHIAN, P.; GROTE, M.; ISARATANAN, W. et al. Oral Manifestations in HIV-positive Adults From Northern Thailand. J Oral Pathol Med, Copenhagen, v.30, n.4, p.220-223, Apr. 2001.

KOSTIALA, I., KOSTIALA, A.A.I., KAHANPÄÄ, A. Oral Mycosis and Their Treatment. Acta Odontol Scand, Stockholm, v.37, n.2, p.87-101, 1979.

LAMEY, P.-J.; LEWIS, M. A. O. A Clinical Guide to Oral Medicine. 2. ed. London: British Dental Association, 1997. 87p.

McCARTHY, G.M.; MACKIE, I.D.; KOVAL, J. et al. Factors Associated With Increased Frequency of HIV-Related Oral Candidiasis. J Oral Pathol Med, Copenhagen, v.20, n.7, p.332-336, Aug. 1991.

MIOTTO, N.M.L., YURGEL, L.S., CHERUBINI, K. Candidíase Oral em Pacientes do Serviço de Estomatologia do Hospital São Lucas da PUCRS. Odonto Ciência, Porto Alegre, v.17, n.38, p.354-361, out/dez. 2003.

NEVILLE, B.W.; DAMM, D.D.; ALLEN, C.M. et al.Patologia Oral e Maxilofacial. Rio de Janeiro: Guanabara Koogan, 1998. 705p.

OKSALA, E. Factors Predisposing to Oral Yeast Infections. Acta Odontol Scand, Stockholm, v.48, n.1, p.71-74, Feb.
1990.

OLIVEIRA, E. E.; SILVA, S. C.; SOARES, A. J. et al. Toxinas Killer e Produção de Enzimas por Candida Albicans Isoladas da Mucosa Bucal de Pacientes com Câncer. Revista da Sociedade Brasileira de Medicina Tropical, v. 31, n. 6 , p. 523-527, nov/dez. 1998.

PUGLIESE, N. S.; ARAUJO, V. C.; BIRMAN, E. G. et al. Prevalência da Língua Geográfica, Língua Pilosa e da Glossite Rômbica em Escolares do $\mathrm{Mu}$ nicípio de São Paulo. Rev Fac Odontol Univ São Paulo, São Paulo, v. 19, n.2, p. 139-142, jul/dez. 1972.

RADFORD, D.R., CHALlaCOMBE, S.J., WALTER, J.D. Denture Plaque and Adherence of Candida Albicans to Denture-base Materials in Vivo and in Vitro. Crit Rev Oral Biol Med, Boca Raton, v.10, n.1, p.99-116, 1999.

RAMIREZ-AMADOR, V., SILVERMAN, S.Jr., MAYER, P. et al. Candidal Colonization and Oral Candidiasis in Patients Undergoing Oral and Pharyngeal Radiation Therapy. Oral Surg Oral Med Oral Pathol Oral Radiol Endod, St Louis, v. 84, n. 2, p. 149-153, Aug. 1997.

RENNIE, J. S.; HUTCHEON, A. W.; MACFARLANE, T. W. et al.The Role of Iron Deficiency in ExperimentallyInduced Oral Candidosis in the Rat. J Med Microbiol, London, v. 16, n. 3, p. 363-369, Aug. 1983.

SHAFER, W.G.; HINE, M.K.; LEVY, B.M. Tratado de Patologia Bucal. 4 ed. Rio de Janeiro: Guanabara Koogan, 1987. $837 p$.

SONIS, S.T.; FAZIO, R.C.; FANG, L. Medicina Oral. Rio de Janeiro: Interamericana, 1985. 497p.

TORRES, S.R.; PEIXOTO, C.B.; CALDAS, D.M. et al. Relationship Between Salivary Flow Rates and Candida Counts in Subjects with Xerostomia. Oral Surg Oral Med Oral Pathol Oral Radiol Endod, St Louis, v.93, n.2, p.149-154, Feb. 2002 .

Endereço para Correspondência: Serviço de Estomatologia do Hospital São Lucas da PUC

Av. Ipiranga, 6690 - Sala 231

POA - RS

Cep:90610-000

E-mail:mazfig@terra.com.br 
Gráfico 1 - FREQÜENCIA DAS FORMAS CLÍNICAS DE CANDIDÍASE BUCAL. PORTO ALEGRE, 2003

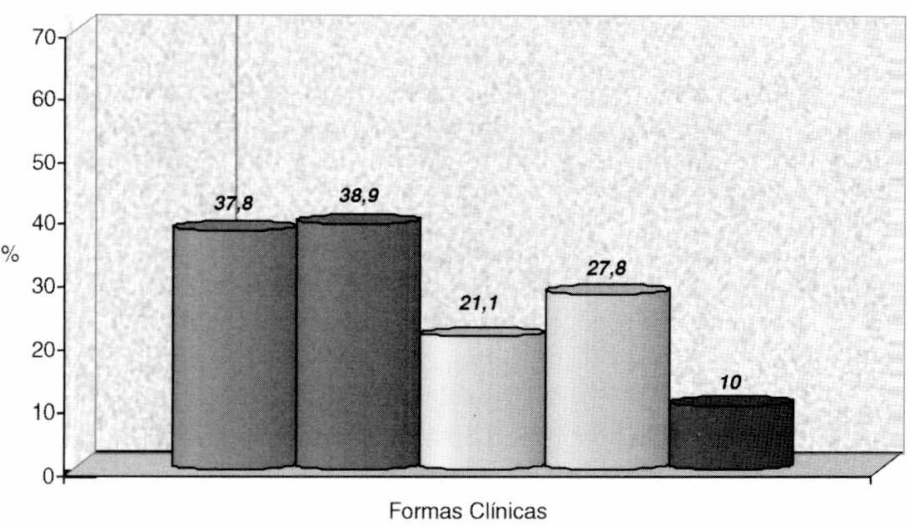

$\square$ Pseudomembranosa $\square$ Eritematosa $\square$ Q. Angular $\square$ Leucoplásica $\square$ GRM

Tabela 2 - FREQUÊNCIA DE CANDIDÍASE ERITEMATOSA DE ACORDO COM OS FATORES AVALIADOS. PORTO ALE-

\begin{tabular}{lccc}
\hline Fatores & Sim & Não & $\boldsymbol{p}$ \\
\hline Tabagismo & $14(40,0 \%)$ & $21(60,0 \%)$ & $0,01^{* *}$ \\
Alcoolismo & $5(14,3 \%)$ & $30(85,7 \%)$ & 0,61 \\
Anemia & $\mathbf{8}(22,9 \%)$ & $27(77,1 \%)$ & $0,04^{*}$ \\
Diabete & $3(8,6 \%)$ & $32(91,4 \%)$ & 0,29 \\
Antibioticoterapia & $3(8,6 \%)$ & $32(91,4 \%)$ & 0,62 \\
Corticoterapia & $6(17,1 \%)$ & $29(82,9 \%)$ & 0,08 \\
Radioterapia & $1(2,9 \%)$ & $34(97,1 \%)$ & 0,67 \\
Quimioterapia & $1(2,9 \%)$ & $34(97,1 \%)$ & 0,49 \\
HIV+ & $9(25,7 \%)$ & $26(74,3 \%)$ & 0,15 \\
Sem fatores associados & $9(25,7 \%)$ & $26(74,3 \%)$ & 0,15
\end{tabular}

* Estatisticamente significativo ( $\mathrm{p} \leq 0,05)$

** Associação negativa
Tabela 1 - FREQUENNCIA DE CANDIDÍASE PSEUDOMEMBRANOSA DE ACORDO COM OS FATORES AVALIADOS. PORTO ALEGRE, 2003

\begin{tabular}{lccc}
\hline Fatores & Sim & Não & $\boldsymbol{p}$ \\
\hline Tabagismo & $17(50,0 \%)$ & $17(50,0 \%)$ & 0,13 \\
Alcoolismo & $7(20,6 \%)$ & $27(79,4 \%)$ & 0,16 \\
Anemia & $5(14,7 \%)$ & $29(85,3 \%)$ & 0,50 \\
Diabete & - & $34(100 \%)$ & 0,09 \\
Antibioticoterapia & $8(23,5 \%)$ & $26(76,5 \%)$ & $0,01 *$ \\
Corticoterapia & $3(8,8 \%)$ & $31(91,2 \%)$ & 0,54 \\
Radioterapia & $3(8,8 \%)$ & $31(91,2 \%)$ & $0,05^{*}$ \\
Quimioterapia & $4(11,8 \%)$ & $30(88,2 \%)$ & $0,02^{*}$ \\
HIV+ & $14(41,2 \%)$ & $20(58,8 \%)$ & $0,01^{*}$ \\
Sem fatores associados & $7(20,6 \%)$ & $27(79,4 \%)$ & 0,48
\end{tabular}

* Estatisticamente significativo $(\mathrm{p} \leq 0,05)$
Tabela 3 - FREQUÊNCIA DE CANDIDÍASE LEUCOPLÁSICA DE ACORDO COM OS FATORES AVALIADOS. PORTO ALE-

\begin{tabular}{lccc}
\hline Fatores & Sim & Não & $p$ \\
\hline Tabagismo & $24(96,0 \%)$ & $1(4,0 \%)$ & $0,01^{*}$ \\
Alcoolismo & $4(16,0 \%)$ & $21(84,0 \%)$ & 0,51 \\
Anemia & - & $25(100 \%)$ & $0,01^{* *}$ \\
Diabete & $1(4,0 \%)$ & $24(96,0 \%)$ & 0,57 \\
Antibioticoterapia & $1(4,0 \%)$ & $24(96,0 \%)$ & 0,29 \\
Corticoterapia & $1(4,0 \%)$ & $24(96,0 \%)$ & 0,22 \\
Radioterapia & - & $25(100 \%)$ & 0,37 \\
Quimioterapia & - & $25(100 \%)$ & 0,26 \\
HIV+ & - & $25(100 \%)$ & $0,01^{* *}$ \\
Sem fatores associados & - & $25(100 \%)$ & $0,02^{* *}$
\end{tabular}

* Estatisticamente significativo $(\mathrm{p} \leq 0,05)$

** Associação negativa 